Onkologe 2018 $\cdot 24: 942-943$ https://doi.org/10.1007/s00761-018-0489-6 Online publiziert: 23. November 2018 (c) Springer Medizin Verlag GmbH, ein Teil von Springer Nature 2018

CrossMark

Rudolf M. Huber ${ }^{1}$ Peter M. Schlag ${ }^{2}$

'Lungentumorzentrum München, LMU Klinikum, Universität München, München, Deutschland

${ }^{2}$ Berlin, Deutschland

\title{
Relevanter Fortschritt beim nichtkleinzelligen Lungenkarzinom
}

\section{Translationale Forschung plus klinische Studien}

In den letzten Jahren hat sich auf allen Gebieten des nichtkleinzelligen Lungenkarzinoms („non-small cell lung cancer", NSCLC) einiges getan. Dies betrifft sowohl Ansätze zur Früherkennung als auch die Verbesserung der Diagnostik und präzisere Therapiemöglichkeiten in deutlich größerer Zahl.

Mit der Computertomographie (CT) des Thorax in Niedrigdosistechnik wurde in einer großen randomisierten PhaseIII-Studie (National Lung Screening Trial, NLST) in den USA gezeigt, dass mit dem Screening von Risikopatienten ein Überlebensvorteil erzielt werden kann. Diese Studie hat zur Empfehlung des Screenings mit Niedrigdosis-CT durch praktisch alle Fachgesellschaften und amtlichen Stellen in den USA geführt. In Europa ist bis jetzt dieses Screening unter kontrollierten Bedingungen möglich, aber nicht flächendeckend empfohlen.

\section{》) Mit dem Screening von Risikopatienten kann ein Überlebensvorteil erzielt werden}

In der Diagnostik des NSCLC haben sich Fortschritte in der Bildgebung mittels PositronenemissionstomographieComputertomographie (PET-CT) und endobronchialem Ultraschall in der Routine ergeben. Damit ist ein exakteres Staging des Mediastinums möglich geworden und können nutzlose Operationen vermieden werden. Hierauf wird in dem Beitrag von S. Kurz und C. Grohé eingegangen. Parallel dazu wurde das TNM-System zweimal aktualisiert und bildet inzwischen die Prognose in den frühen Stadien und in der Metastasierung deutlich besser ab; dies hat u. a. auch zu einer konzeptionellen Präzisierung der Behandlung bei Patienten mit oligometastasierten Tumoren beigetragen.

Wichtige Fortschritte wurden aber v. a. auch in der molekularpathologischen Diagnostik erzielt. In der Regel ist daher neben der bisher alleinigen Unterteilung des NSCLC in die verschiedenen histologischen Typen und Subtypen des Adenokarzinoms eine zusätzliche molekularbiologische Analyse $\mathrm{zu}$ fordern. Aktuell sollten sich die molekularen Analysen insbesondere auf EGFR-, BRAF-p.V600-Mutationen sowie ALK- oder ROS1-Translokationen beziehen, da sich daraus relevante therapeutische Konsequenzen ergeben können. Für die Therapie mit Immuncheckpointinhibitoren ist der Nachweis von PD-L1 von Relevanz. Alle wichtigen Zusammenhänge und Details sind dem Artikel von M. Wittersheim et al. zu entnehmen.

In der Therapie wurden Fortschritte sowohl im operablen Stadium als auch im lokal fortgeschrittenen Stadium erzielt. Die systemische Therapie wurde fester Bestandteil in multimodalen Konzepten. Die bereits kurz skizzierten molekularbiologischen Erkenntnisse, insbesondere die für die Tumorigenese wichtigen „Treibermutationen“, haben für einen relevanten Teil der Patienten im metastasierten Stadium die Therapielandschaft nachhaltig verändert und verbessert. Hierauf wird ausführlich im Beitrag von S. Michels und J. Wolf eingegangen. Dabei wird auch aufgezeigt, dass die die Suche nach weiteren onkogenen Aberrationen zur Aufdeckung zusätzlicher, z. T. sehr kleiner genetischer Subgruppen geführt hat. Hierzu zählen Amplifikationen, Mutationen sowie Translokationen im MET-RezeptorGen, eine Translokation im NTRK1/2/3Gen sowie RET-Translokationen. Es ist zu erwarten, dass durch auf diese Targets entwickelte und bereits in klinischer Prüfung befindliche Medikamente die Behandlung des NSCLC weiter präzisiert und verbessert werden kann.

\section{》) Die meisten Resektionen erfolgen in den nicht lokal fortgeschrittenen Stadien thorakoskopisch}

In der Chirurgie war bis vor wenigen Jahren die offene Thorakotomie Standard. Inzwischen werden die meisten Resektionen in den nicht lokal fortgeschrittenen Stadien thorakoskopisch durchgeführt mit geringerer Belastung für die Patienten. Diese Technik stellen L. Klotz und H. Winter im Artikel über die operative Therapie im Stadium I dar. Ebenfalls in den letzten Jahren hat sich die im Beitrag von J. Fleckenstein erläuterte stereo- 
taktische Strahlentherapie des peripheren Rundherds als relevante Alternative (zumindest bei den funktionell inoperablen Patienten) etabliert. Für 2 randomisierte Studien verlief die Rekrutierung jedoch so schlecht, dass sie geschlossen werden mussten. In diesen Studien sollte bei funktionell inoperablen Patienten die thorakoskopische Resektion gegenüber der stereotaktischen Strahlentherapie geprüft werden. Damit kann bei operablen Patienten im Stadium I keine verlässliche Aussage gemacht werden, welche Methodik bei welchen Patienten am besten einzusetzen ist.

Im Stadium III wird zwischen funktionell und technisch sowie onkologisch operablen und inoperablen Tumoren unterschieden. In der Strahlentherapie war noch nicht flächendeckend die Radiochemotherapie etabliert. Die simultane Chemotherapie ist nun in der Regel Standard für das lokal fortgeschrittene und inoperable nichtkleinzellige Lungenkarzinom. Im Jahr 2017 hat sich in einer Studie der Phase III gezeigt, dass eine adjuvante Immuncheckpointinhibition die Prognose der Patienten mit lokal fortgeschrittenem NSCLC verbessert. Diese Entwicklung wird in dem Artikel zum Stadium III des nichtkleinzelligen Lungenkarzinoms von W. Eberhardt et al. erörtert. Im operablen Stadium wird in der Regel eine adjuvante oder auch neoadjuvante Chemotherapie durchgeführt. Abhängig vom Ausmaß des mediastinalen Lymphknotenbefalls erfolgt zusätzlich eine perkutane mediastinale Bestrahlung. Auch Ansätze zur primären trimodalen Therapie im Stadium III sind weiter aktuell.

Im Stadium IV sollte als Einzelfallentscheidung im Tumorboard bei einer oligometastatischen Erkrankung geprüft werden, ob mit einer multimodalen Therapie ein kurativer Ansatz besteht. Darauf wird im Beitrag F. Griesinger et al. näher eingegangen. Patienten mit Treibermutationen sollten eine entsprechend zielgerichtete Therapie erhalten. Falls bei Fehlen einer Treibermutation eine PD-L1Expression von über $50 \%$ vorliegt, wird eine Therapie mit Pembrolizumab, einem PD-L1-Inhibitor, eingeleitet. Bei den restlichen NSCLC haben Studien einen Vorteil der Kombination einer Chemothera- pie mit Immuncheckpointinhibition gezeigt. Eine Zulassung für den europäischen Markt liegt jedoch bis jetzt noch nicht vor. Auch nach Versagen der Erstlinientherapie haben sich in den letzten Jahren die Optionen kontinuierlich erweitert, wie im abschließenden Beitrag von A. Rittmeyer ausführlich dargestellt wird.

Diese kurzen Ausführungen mögen veranschaulichen, dass bei einem Tumor wie dem NSCLC, der sehr lange mit einer äußerst schlechten Prognose behaftet war, durch konsequente Forschung in Verbindung mit adäquaten klinischen Studien erfreuliche Fortschritte erzielt worden sind.

Für die Schriftleiter

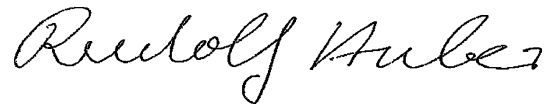

R. M. Huber

Für die Herausgeber

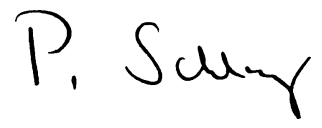

P. M. Schlag

\section{Korrespondenzadresse}

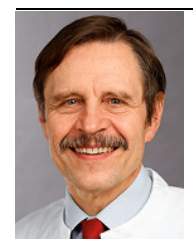

Prof. Dr. Rudolf M. Huber

Lungentumorzentrum

München, LMU Klinikum,

Universität München

München, Deutschland pneumologie@med.unimuenchen.de

Interessenkonflikt. R.M. Huber gibt an, dass klinische Studien und Vorträge zu den angeführten Medikamenten und Therapien durchgeführt wurden und werden. P.M. Schlag gibt an, dass kein Interessenkonflikt besteht.

\section{ONKO-Internetportal}

Neues von den Kongressen ESMO und DGHO

Im Herbst ist die Kongressdichte für Onkologen und onkologisch tätige Ärzte besonders hoch. In jährlichem Rhythmus geben Tagungen der deutschen und europäischen Fachgesellschaften einen umfassenden Überblick über den aktuellen Stand der Krebsforschung. Der größte europäische Krebskongress, die Jahrestagung der European Society for Medical Oncology (ESMO), fand in diesem Jahr in München statt.

Das Redaktionsteam des ONKO-Internetportals war vor Ort und liefert unter www.krebsgesellschaft.de/esmo2018 Expertenmeinungen zu den wichtigsten Themen aus erster Hand. Diskutiert werden unter anderem die Überlebensdaten der PACIFIC-Studie und deren mögliche Auswirkungen auf die Behandlungsabläufe von Patienten mit inoperablem Lungenkrebs sowie vielversprechende Daten im Bereich der Immuntherapie beim Mammakarzinom. Thema sind außerdem die Updates der drei Phase-III-Studien PROSPER, SPARTAN und LATITUDE, die starken Einfluss auf die Therapieentscheidungen beim Prostatakarzinom haben.

Aktuelle Impressionen von der diesjährigen Jahrestagung der Deutschen, Österreichischen und Schweizerischen Gesellschaften für Hämatologie und Medizinische Onkologie (DGHO) liefert das ONKO-Internetportal unter www.krebsgesellschaft.de/dgho2018. Themenschwerpunkte in den Experteninterviews sind neben modernen Therapieansätzen aus den Bereichen Immuntherapie und Stammzellentransplantation insbesondere auch neue Daten aus der Grundlagen-, der angewandten und translationalen Forschung.

$$
\begin{array}{r}
\text { Quelle } \\
\text { ONKO-Internetportal } \\
\text { in Kooperation mit der } \\
\text { Deutschen Krebsgesellschaft e.V. } \\
\text { (DKG) } \\
\text { www.krebsgesellschaft.de }
\end{array}
$$

